Check for updates

Cite this: RSC Adv., 2019, 9, 8537

Received 5th January 2019

Accepted 20th February 2019

DOI: 10.1039/c9ra00106a

rsc.li/rsc-advances

\section{Neodymium-decorated graphene oxide as a corrosion barrier layer on Ti6Al4V alloy in acidic medium $\dagger$}

\author{
N. Palaniappan, (D)*a I. S. Cole, (D)*b F. Caballero-Briones, (D) ${ }^{c}$ S. Manickam, (iD ${ }^{d}$ C. Lal ${ }^{e}$ \\ and J. Sathiskumar ${ }^{f}$
}

Ti6Al4V alloy is light weight and is used in construction, oil industries and airbus, automobile, and bio implant materials. The native oxide layers of the alloy are not stable at high temperatures and strong mineral acid environments. The conventional epoxy-based layers are porous and the alloy finally fails in the harsh environment in the long term. Therefore, the carbon-based functional materials are being proposed as coating materials to overcome the alloy degradation. In the present contribution, we have used the neodymium-decorated graphene oxide as the corrosion inhibiting barrier for the Ti6Al4V alloy. As a novelty, we found that the few-layer graphene decorated with neodymium acts as a self-cleaning coating. The Nd-decorated graphene oxide were studied by XRD, TEM, FESEM, FTIR, UV, and Raman spectroscopy. The corrosion inhibition efficiency was studied by electrochemical methods.

\section{Introduction}

Recently titanium-based alloys, specifically Ti6Al4V, have been used to prepare body parts in airbus, automobile industry, nuclear fuel plants and bio-implants due to their strong corrosion resistance in the environment. In the past decades, epoxy coating has been used for corrosion inhibition in the acidic and salt environments. The problem of epoxy coating is the large pore size that allows the ions to penetrate, letting the alloy surface be damaged in the long term. Early reports have shown that Pd-, Pt-, and Ru-based titanium alloys have a strong corrosion resistance in the acidic environment, ${ }^{1}$ but noble metals are economically unfavourable for making the industrial components. Moreover, several authors reported that the hydroxyl derivatives and heterocyclic compounds can act as corrosion inhibitors in the acidic and salt media. ${ }^{2,3}$ However, the organic inhibitors do not achieve long-term corrosion inhibition efficiency in the acidic media. Hence, the carbonbased functional materials have been proposed to overcome

${ }^{a}$ School of Chemical Sciences, Central University of Gujarat, 382030, India. E-mail: palaniappancecri@rediffmail.com

${ }^{b}$ Advance Manufacturing and Fabrication Research and Innovation, RMIT University, Melbourne, Vic 3100, Australia

'Instituto Politecnico Nacional, Materials and Technologies for Energy, Health and Environment (GESMAT), CICATA Altamira, 89600, Altamira, Mexico

${ }^{d}$ Faculty of Science and Engineering, University of Nottingham Malaysia, Jalan Broga 43500, Malaysia

${ }^{e}$ Department of Chemistry, Harcourt Butler Technical University, Kanpur, India

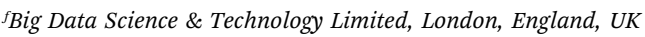

$\dagger$ Electronic supplementary information (ESI) available. See DOI: $10.1039 / \mathrm{c} 9 \mathrm{ra00106a}$ the long-term corrosion inhibition failure in the acidic medium. ${ }^{4}$ The carbon-based materials can achieve excellent adsorption on the metal surface, which allows this family of materials to act as corrosion inhibition barrier in several environments. Recently, graphene oxide-functional materials are becoming significant for surface protection because the electrolytes are not able to penetrate through the layered structure of graphene oxide. ${ }^{5}$ Singhbabu et al. reported a green, oil-based graphene oxide corrosion inhibitor for mild steel, whose performance increased as it improved the hydrophobicity of the coated surface. ${ }^{6}$ Dutta et al. reported a graphene oxide-polymer composite material that reinforced the corrosion inhibition in the mild steel, which was attributed to the improved hydrophobicity due to the polymer matrix. ${ }^{7}$ Furthermore, Huh et al. reported that the acetone-derived graphene oxide acts as a corrosion inhibitor on copper in a $3.5 \% \mathrm{NaCl}$ medium. ${ }^{8}$ Maia et al. reported that silica nano particles have been used as a corrosion barrier for an aluminium alloy in salt environments. ${ }^{9}$ Liu et al. reported the investigation of an imidazole-based ionic liquid functionalized graphene oxide as a corrosion inhibitor in $\mathrm{NaCl}$ solution on the mild steel; ${ }^{10}$ the authors attributed the enhanced corrosion inhibition efficiency to the improved electron transfer between the coating surface and the aggressive ions, favoured by the anionic and cationic parts of the ionic liquids. In addition, several authors reported the corrosion inhibition barrier layers in the $3.5 \% \mathrm{NaCl}$ environment, prepared from graphene oxides functionalized with pyridine as well as composites of graphene oxide with polystyrene, metronidazole, polyvinylene and lignin. ${ }^{11-18}$ Moreover, the rare earth elements have been reported as green corrosion inhibitors because of their biocompatibility and the ability to 
transfer electrons to the vacant metal d orbitals. It is also known that the lanthanide series elements can improve the long-term corrosion inhibition efficiency because they have multiple oxidation states. Several authors have reported lanthanide series composite materials and additives for various alloys to improve their corrosion resistance in salt environments. The lanthanides such as $\mathrm{Nd}, \mathrm{Gd}$ and Ce have been recently found to improve the corrosion resistance in acidic environments. ${ }^{\mathbf{1 9 - 2 3}}$ The addition of $\mathrm{La}, \mathrm{Sm}$, Tb or Dy as a microstructure filler to different alloys for the enhanced corrosion resistance has been also investigated. In other reports, $\mathrm{La}^{3+}$ was incorporated into the polymer matrix and Ce-modified coating, $\mathrm{Nd}$ and Ce additives, imidazole derivatives and $\mathrm{N}$-functionalized graphene oxide have been demonstrated to improve the corrosion resistance in a $3.5 \% \mathrm{NaCl}$ solution. ${ }^{24-31}$ Recently, we investigated the praseodymium-decorated graphene oxide as an inhibition barrier layer on magnesium AZ13 alloy in strong acidic environments, and showed that GO-Pr improved the inhibition efficiency because the corrosive species could not interact with the alloy surface. ${ }^{32}$ Even though the corrosion inhibition activity of the neodymium-decorated graphene oxide has not been studied to the best of our knowledge, their slightly minor ionic ratio $\left(\mathrm{Nd}^{3+} 112 \mathrm{pm}, \mathrm{Pr}^{3+} 112 \mathrm{pm}\right)$ and the presence of the $2^{+}$and $3^{+}$states in comparison with Pr that preferentially displays the $3^{+}$state could be beneficial for the redox-aided inhibition as mentioned above. Thus, in the present work, we investigated the effect of a neodymium-decorated graphene oxide layer to assess its corrosion inhibition activity in acidic environments in the Ti6Al4V alloy.

\section{Experimental}

Natural graphite powder, sodium nitrate, $\mathrm{H}_{2} \mathrm{O}_{2}, \mathrm{KMnO}_{4}$, and neodymium(III) nitrate hexahydrate $\left(\mathrm{Nd}\left(\mathrm{NO}_{3}\right)_{3} \cdot 6 \mathrm{H}_{2} \mathrm{O}\right)$ were purchased from Alfa Aesar and used without further purification. The GO synthesis was carried out by the modified Hummers method. According to our previous work, ${ }^{32} 1 \mathrm{~g}$ of natural graphite powder, $50 \mathrm{~mL}$ of $\mathrm{H}_{2} \mathrm{SO}_{4}$, and $40 \mathrm{~mL}$ of $\mathrm{H}_{3} \mathrm{PO}_{4}$ were added into a $250 \mathrm{~mL}$ round bottom flask and the flask was kept in an ice container, followed by the slow addition of $3 \mathrm{~g}$ of the oxidation agent $\mathrm{KMnO}_{4}$ to the mixture. The mixture temperature was increased and kept at $60{ }^{\circ} \mathrm{C}$ during $12 \mathrm{~h}$ of reflux. Afterwards, the mixture was allowed to cool to room temperature and then poured into $1000 \mathrm{~mL}$ of ice water for fine dispersion. Then, $10 \mathrm{~mL}$ of $\mathrm{H}_{2} \mathrm{O}_{2}$ was added to the mixture to terminate the reaction. The product was washed three times with $1 \mathrm{M} \mathrm{HCl}$ for the removal of reaction by-products. The GO was washed several times with distilled water till the solution became neutral. The final product was dried at $80{ }^{\circ} \mathrm{C}$ in a vacuum oven.

\subsection{GO-neodymium decoration}

For decoration, $100 \mathrm{mg}$ of neodymium(III) nitrate hexahydrate and $200 \mathrm{mg}$ of graphene oxide were mixed in a round bottom flask containing $100 \mathrm{~mL}$ of distilled water. The mixture was sonicated for $1 \mathrm{~h}$ for fine dispersion in a SB-300DTYMULI
Ultrasonic cleaner. Then, $10 \mathrm{~mL}$ of $1 \mathrm{M} \mathrm{NaOH}$ was added to the mixture as an activator. The mixture was stirred for $24 \mathrm{~h}$ at room temperature. The product was washed with distilled water until a neutral solution was obtained. The $\mathrm{GO}+\mathrm{Nd}$ final product was dried at $80^{\circ} \mathrm{C}$ in a vacuum oven.

\subsection{Material characterization}

The UV-visible absorption measurements were performed on a Shimadzu UV-VIS 3600 spectrometer. The molecular structure of the prepared materials was studied by the $\mathrm{KBr}$ pellet method on a Spectrum 65 Fourier Transform Infrared Spectrometer (FTIR) from Perkin Elmer. The Raman spectra were acquired from a WITec confocal Raman spectrometer, using a $532 \mathrm{~nm}$ laser as the excitation source. The crystal structure was studied by X-ray diffraction analysis performed on a D8 Advance diffractometer from Bruker, using $\mathrm{Cu} \mathrm{K} \alpha$ radiation $(\lambda=1.5406$ $\AA$ ) in the Bragg-Brentano geometry. The morphology was analysed by a Carl Zeiss FESEM Marline microscope. The microstructure of graphene oxide and neodymium-decorated graphene oxide were confirmed by the Transmission Electron Microscopy (TEM) on a FEI Techani G2 Twin 500. The coated films on the Ti6Al4V alloy surface were studied by Attenuated Total Reflectance (ATR) on a Thermo Fisher ATR-FTIR iD7 Nicolet S5 Model spectrometer. The coating thickness was analysed by ellipsometry, with the incident light at $70^{\circ}$ and in a range from 0.6500 to $6.500 \mathrm{eV}$ with $0.05 \mathrm{eV}$ increment on a HORIBA UVI Cell2 Ellipsometer.

The theoretical study to model the interactions between the $\mathrm{GO}+\mathrm{Nd}$ coating and the Ti6Al4V surface to assess the corrosion inhibition activity was carried out using a 03 Gaussian programme package B3LYP $312 * \mathrm{G}$ basis set solvent methods. The electronegativity $(\chi)$, chemical hardness $(\eta)$, softness $(\sigma)$, electrophilicity $(\omega)$, and quantum chemical parameters were calculated by following equations:

$$
\begin{gathered}
\chi=\frac{I+A}{2} \\
\eta=\frac{I-A}{2} \\
\sigma \frac{1}{\eta} \\
\omega=\frac{\chi^{2}}{2 \eta}
\end{gathered}
$$

\subsection{Corrosion study}

The titanium Ti6Al4V alloy was cut into $1 \mathrm{~cm} \times 1 \mathrm{~cm}$ pieces. The alloy was connected with a copper wire for electrical conduction and was mounted with cold cure material on one side to block the alloy surface. The exposed area of the alloy was polished with emery paper with 600 to 1200 grits, rinsed with soap solution and distilled water, and finally washed with acetone. The material was kept in a desiccator before use. The coating material was prepared with a $1: 2 \mathrm{wt} \%$ ratio of hardener 
(polyamine) epoxy and $\mathrm{GO}+\mathrm{Nd}$, dispersed in ethanol and sonicated for $1 \mathrm{~h}$ in a SB-300DTYMULI ultrasonic cleaner. A colloid solution was formed and used as the coating material. This was carefully applied onto the polished TI6Al4V surface using a $0.2 \mathrm{~mm}$ paint brush. The Ti6Al4V-coated coupons were dried at $80^{\circ} \mathrm{C}$ for $24 \mathrm{~h}$ in a vacuum oven before the corrosion inhibition study. The corrosion inhibition study was done using a CHI 920D electrochemical station and the coated coupons of the TI6Al4V alloy as the working electrode. A platinum foil was used as the counter electrode and an $\mathrm{Ag} / \mathrm{AgCl}$ electrode was used as the reference electrode. The corrosion inhibition efficiency was calculated by the following equation:

$$
\begin{gathered}
\eta=\frac{I_{0}-I_{\mathrm{I}}}{I_{0}} \\
C_{\mathrm{dl}}=\frac{1}{2 \pi f_{\max } R_{\mathrm{ct}}} .
\end{gathered}
$$

\section{Results and discussion}

\subsection{UV-vis and infrared characterization}

The optical absorption spectra of GO and GO + Nd are shown in Fig. 1. The optical absorption has been used to investigate the electronic transitions of GO and GO + Nd composite materials. The GO absorbs in the range from $200 \mathrm{~nm}$ to $250 \mathrm{~nm}$, which is attributable to the electronic transition between $\pi$ and $\pi^{*} .{ }^{33,34}$ However, GO + Nd spectrum presents sharp peaks between 300 and $350 \mathrm{~nm}$ due to the $\mathrm{n}-\pi$ electron transition in neodymium. The further absorption peaks observed in $\mathrm{GO}+\mathrm{Nd}$ spectrum at $500 \mathrm{~nm}, 650 \mathrm{~nm}, 750 \mathrm{~nm}, 800 \mathrm{~nm}$, and $900 \mathrm{~nm}$ are due to the valence electron transitions in neodymium. ${ }^{35}$

Fig. 2 presents the FTIR spectra of GO and $\mathrm{GO}+\mathrm{Nd}$ obtained by the $\mathrm{KBr}$ pellet method. The GO spectrum displays features related with the carboxyl group's stretching frequency that appears around $1740 \mathrm{~cm}^{-1} \cdot{ }^{36}$ The GO carbonyl groups stretching frequency is around $1060 \mathrm{~cm}^{-1}$, while the edge hydroxyl groups stretching frequency is around $1265 \mathrm{~cm}^{-1}$. Correspondingly, the FTIR spectrum of the neodymium-decorated graphene oxide presents the bond stretching frequency at $1600 \mathrm{~cm}^{-1}$ related with the $\mathrm{C}=\mathrm{C}$ bond, ${ }^{37}$ suggesting a strong

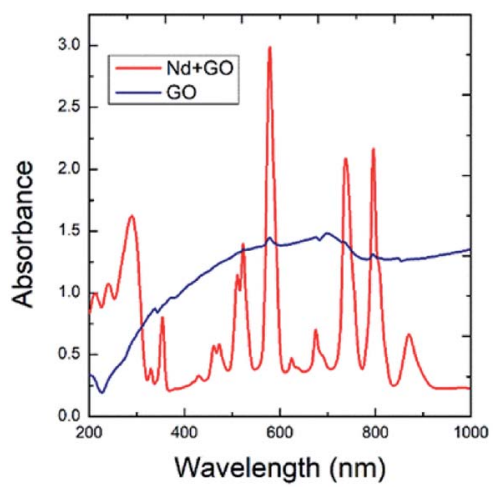

Fig. 1 Optical absorption spectra of $\mathrm{GO}$ and $\mathrm{GO}+\mathrm{Nd}$ materials.

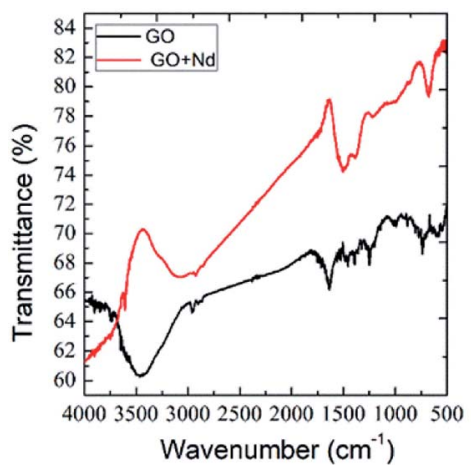

Fig. 2 FTIR spectra of $\mathrm{GO}$ and $\mathrm{GO}+\mathrm{Nd}$.

reduction of GO upon decoration, as reported before for Prdoped $\mathrm{GO}^{32}$

\subsection{Structure}

The Raman spectra of the prepared materials are shown in Fig. 3A. The GO spectrum features the characteristic D, G and $2 \mathrm{G}$ bands attributed to the disorder originating from the $\mathrm{sp}^{3}$ peaks, carbon symmetric vibrations and high order vibrations of the carbon skeleton ${ }^{43}$ at around $1360 \mathrm{~cm}^{-1}, 1580 \mathrm{~cm}^{-1}$, and $2600 \mathrm{~cm}^{-1}$, respectively. $\mathrm{GO}+\mathrm{Nd}$ spectrum presents the same bands with a noticeable higher intensity $\mathrm{G}$ band as well as a peak at around $780 \mathrm{~cm}^{-1}$ related to $\mathrm{Nd}(\mathrm{OH})_{3}$ nanorods, corroborating the strong GO reduction suggested by the results from FTIR spectroscopy. ${ }^{\mathbf{4 4 , 4 5}}$

The X-ray diffractograms of the neodymium-decorated graphene oxide and GO are shown in Fig. 3B. The GO diffractogram shows a peak at $2 \theta=15^{\circ}$ associated with the GO (002) plane and a peak at $2 \theta=25^{\circ}$ attributed to the graphitic carbon. In the diffractogram of $\mathrm{GO}+\mathrm{Nd}$, several peaks were indexed to the (104), (004), (105), (200), (211) and (213) crystal planes of the hexagonal phase of $\mathrm{Nd}(\mathrm{OH})_{3}$ nanorods. ${ }^{38-42}$

\subsection{Morphology and composition}

Fig. 4 displays the morphology study of GO and GO + Nd. Fig. 4A presents a GO micrograph, where the stacked GO sheets can be clearly observed. Fig. 4C presents the corresponding
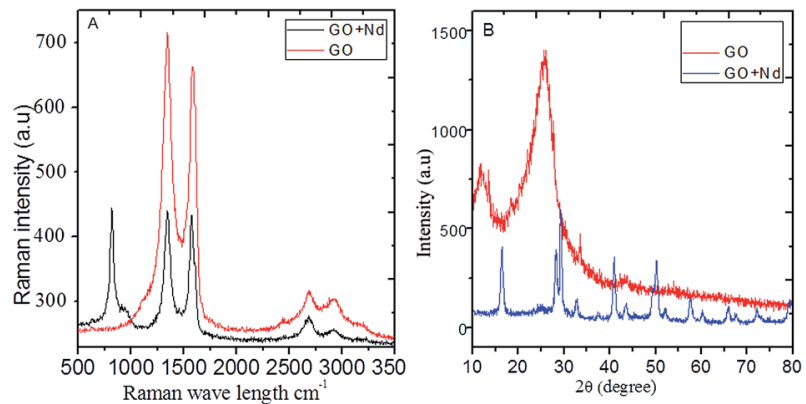

Fig. 3 (A) Raman spectra of $\mathrm{GO}$ and $\mathrm{GO}+\mathrm{Nd}$; (B) X-ray diffractograms of $\mathrm{GO}$ and $\mathrm{GO}+\mathrm{Nd}$ materials. 


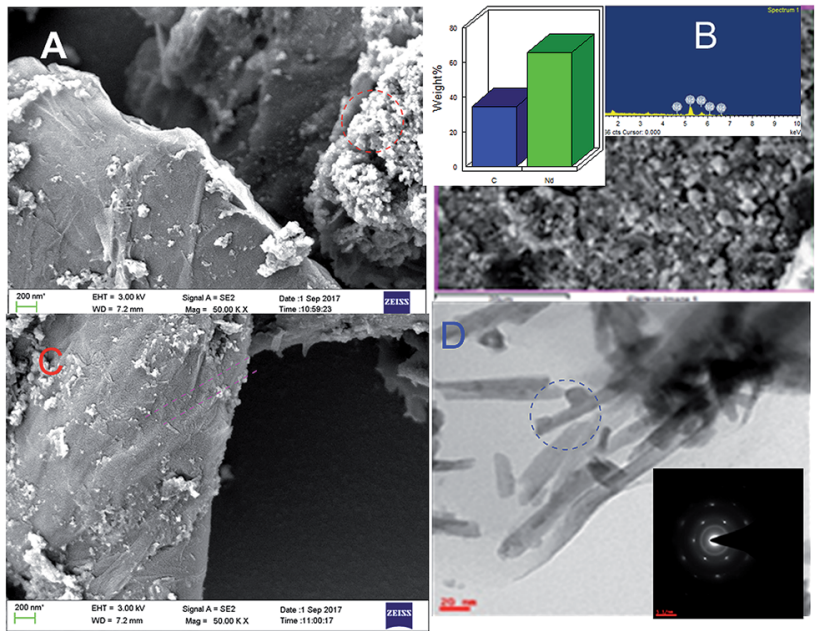

Fig. 4 FESEM micrographs of (A) GO and (C) GO + Nd. (B) FESEM micrograph and EDS spectrum of the GO + Nd sample and (D) TEM micrograph with electron diffraction pattern in the inset.

micrograph of the GO + Nd sample, where it can be observed that the sheets are covered with a powdery-like deposit. Fig. 4B presents the morphology of a selected region and the EDS spectrum, where $\mathrm{Nd}$ can be observed, indicating the $\mathrm{Nd}$ interaction with the graphene oxide sheet. ${ }^{46-50}$ The TEM image presented in Fig. 4D shows the defined rods with a hexagonal structure as inferred from the electron diffraction pattern shown in Fig. 4D inset. The morphology and structure agrees with the XRD and Raman data. As shown in Fig. 5, the EDS elemental mapping of $\mathrm{GO}+\mathrm{Nd}$ indicates that $\mathrm{Nd}^{3+}$ cation is uniformly distributed on the graphene oxide sheet.

\subsection{Proposed mechanism for neodymium decoration}

With the experimental evidence of FTIR spectroscopy, Raman spectroscopy, XRD and TEM/FESEM, a mechanism to explain the Nd decoration in GO sheet is proposed. As shown in Fig. 6, $\mathrm{Nd}^{3+}$ in basic media interacts with the negatively charged graphene oxide sheet with the epoxy moieties. ${ }^{51}$ Fig. 7 presents
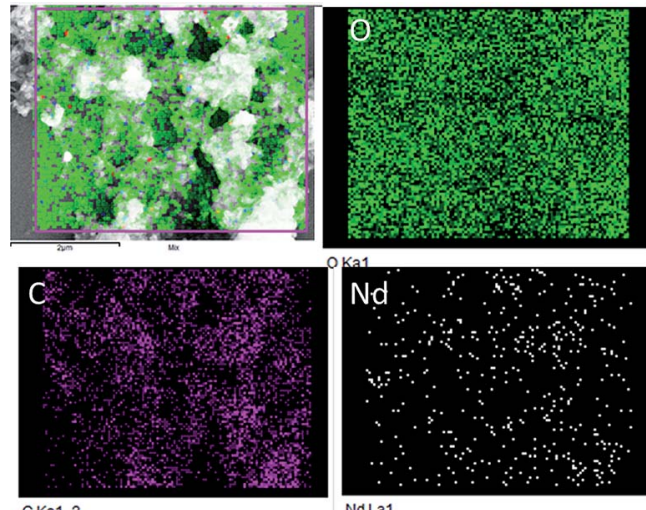

Fig. 5 Elemental mapping of $\mathrm{GO}+\mathrm{Nd}$.

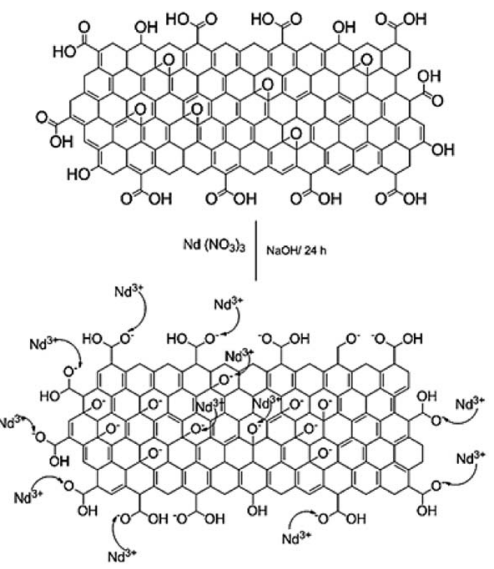

Fig. 6 Proposed reaction mechanism of the reaction between $\mathrm{Nd}^{3+}$ and the graphene oxide epoxy groups.

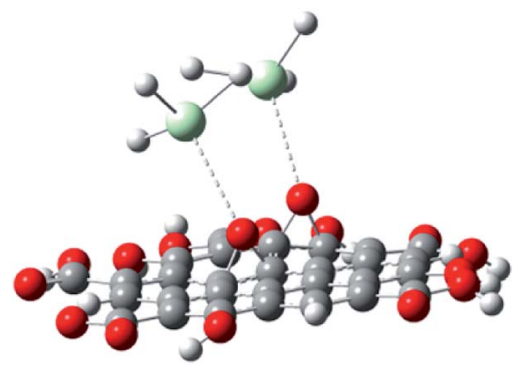

Fig. 7 Neodymium cation coordination with GO epoxy moieties.

a model of interaction between the neodymium ions with carboxyl oxygens. The out-of-the-plane epoxy groups in the graphene oxide sheet could be the most efficient to be coordinated with neodymium $\mathrm{Nd}^{3+}$. Therefore, in the alkaline conditions prevalent during the reaction, the growth of out-ofthe-plane $\mathrm{Nd}(\mathrm{OH})_{3}$ rods is favoured.

\subsection{Redox activity}

Fig. 8 presents the cyclic voltammograms of the $\mathrm{GO}+\mathrm{Nd}$ coating on the Ti6Al4V coupons to study the redox behaviour of the coating using $50 \mu \mathrm{M} \mathrm{K}{ }_{4} \mathrm{Fe}(\mathrm{CN})_{6}$ solution as a probe and $1 \mathrm{M}$ $\mathrm{H}_{2} \mathrm{SO}_{4}$ as an electrolyte. Upon potential cycling, the electrode
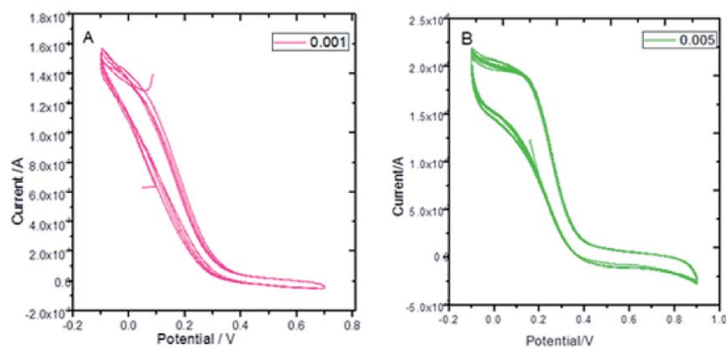

Fig. 8 Cyclic voltammetry of $\mathrm{GO}+\mathrm{Nd}$ at different scan rates: (A) $0.001 \mathrm{mV} \mathrm{s}^{-1}$, (B) $0.005 \mathrm{mV} \mathrm{s}^{-1}$ using a $\mathrm{K}_{4} \mathrm{Fe}(\mathrm{CN})_{6}$ redox probe in $1 \mathrm{M}$ $\mathrm{H}_{2} \mathrm{SO}_{4}$ electrolyte. 
Table 1 Corrosion inhibition efficiency values of epoxy and GO + Nd coated Ti6Al4V alloys and film thickness values

\begin{tabular}{lllllll}
\hline S. no. & $-E_{\text {corr }}(\mathrm{mV})$ & $-I_{\text {corr }}(\mathrm{mA})$ & $(\%)$ & $R_{\text {ct }}\left(\Omega \mathrm{cm}^{2}\right)$ & $C_{\mathrm{dl}} \mu \mathrm{F}$ & $\chi^{2}$ \\
\hline Epoxy & 122 & 6.179 & & 480 & 3.7046 & 1.7149 \\
GO + Nd & 850 & 2.201 & 64.5 & 970 & 2.7333 & 0.7670
\end{tabular}
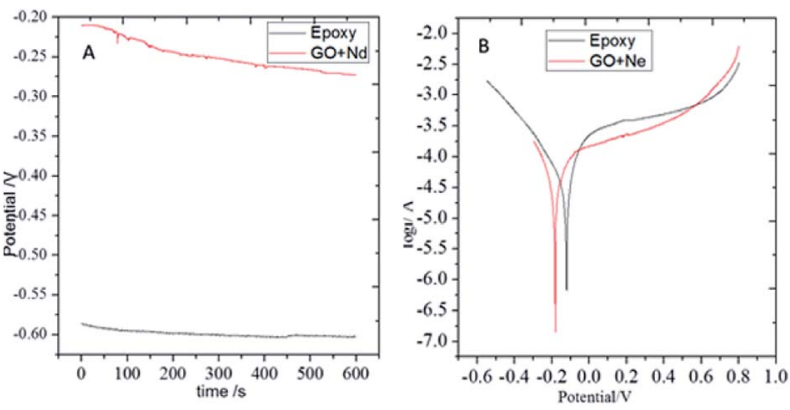

Fig. 9 (A) OCP of epoxy and GO + Nd coated Ti6Al4V alloys and (B) potentiodynamic plots of epoxy and GO $+\mathrm{Nd}$ coated Ti6Al4V alloy.

featured almost no electrochemical response at potentials above $0.4 \mathrm{~V}$, while a large anodic response was evident when the potential was swept between 0.4 and $-0.2 \mathrm{~V}$, suggesting some penetration of the coating. However, the current remained stable during the 5 cycles, demonstrating the coating stability. This indicated that the $\mathrm{GO}+\mathrm{Nd}$ coating is being restored during the cycling, protecting the Ti6Al4V surface. ${ }^{52-55}$ Fig. S1† presents the ellipsometry spectra of the $\mathrm{GO}+\mathrm{Nd}$ coating and the reference epoxy coating. The calculated coating thicknesses from spectrafitting are shown in Table 1. The epoxy and GO + Nd coating thicknesses are $10.952 \mu \mathrm{m}$ and $14.872 \mu \mathrm{m}$, respectively. ${ }^{56-59}$

\subsection{Electrochemical study of corrosion inhibition}

Open circuit potential (OCP) measurements are shown in Fig. 9. The epoxy coating potential has an OCP value of around $-0.6 \mathrm{~V}$, indicating the easiness of corrosion of the alloy. The epoxy coating is porous and the aggressive ions can penetrate to the alloy surface. Moreover, the GO + Nd coating OCP values largely shifted to the less negative potentials with values ranging from -0.20 to $-0.25 \mathrm{~V}$, indicating that the coating does not allow the corrosive ions to penetrate to the alloy surface. ${ }^{60-65}$ In Fig. 9, the potentiodynamic polarization behaviour of the epoxy and GO + Nd coatings are shown. The epoxy coating corrosion potential decreased and the corrosion current increased because of the porosity and poor adhesion of the epoxy coating on the metal alloy surface. ${ }^{66-75}$ Correspondingly, for the $\mathrm{GO}+\mathrm{Nd}$ coated alloy, the cathodic hydrogen evolution was in control and the anodic metal dissolution decreased because of the strong impermeable barrier layer on the alloy, leading to an increase in the corrosion potential and the decrease, by one order of magnitude, in the corrosion current.

As shown in Fig. 10, the Nyquist spectrum of the epoxy coating presents a suppressed semicircle due to pit formation on the alloy surface and coating instability in the acidic environment. ${ }^{69-75}$ In the case of the GO + Nd coating, the Nyquist plot shows an enlarged semicircle because of the barrier layer

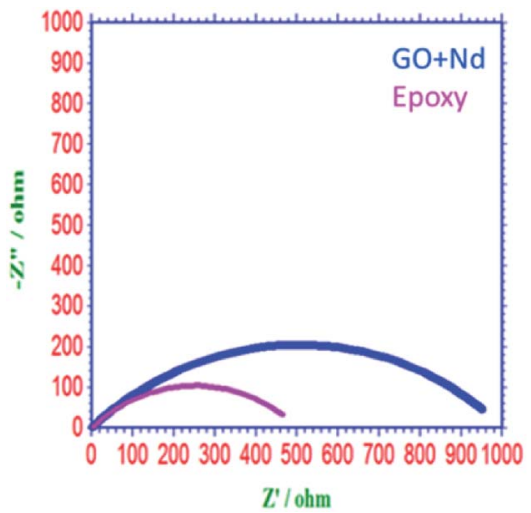

Fig. 10 Nyquist plot of epoxy and GO + Nd-coated Ti6Al4V alloys.

adsorbed on the alloy surface. According to the charge transfer values of GO + Nd coating, the value increased to $980 \Omega$ compared to the $470 \Omega$ of the reference epoxy coating, which is result of the alloy porosity. The double layer capacity of the epoxy coating is higher than that of the $\mathrm{GO}+\mathrm{Nd}$ because the epoxy coating could not control the ion diffusion. ${ }^{30-33}$ The corrosion inhibition efficiency is $64.5 \%$ higher for the GO + Nd coating, demonstrating that it is an excellent corrosion resistance coating material in the acidic environment for the Ti6Al4V alloy.

\subsection{Surface studies of the coated Ti6Al4V alloys}

Fig. 11 presents the morphology of the (A) GO + Nd coated and (B) epoxy coated Ti6Al4V alloys. As shown in Fig. 11, the GO + Nd coating has a homogeneous surface because the barrier layer does not allow corrosive ions into the coating surface, ${ }^{76,77}$ indicating the strong physisorption on the alloy surface of the GO + Nd-based coating. The alloy dissolution has been controlled by the impermeable $\mathrm{GO}+\mathrm{Nd}$ sheet on the alloy surface as shown in the electrochemical characterization. As
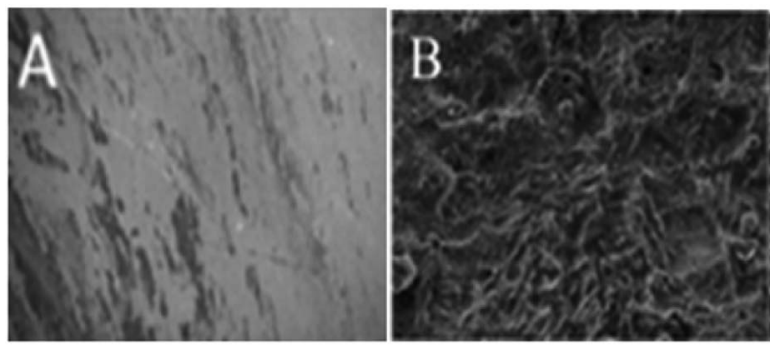

Fig. 11 After corrosion treatment surface morphology of the GO + $\mathrm{Nd}$-coated Ti6Al4V alloy (A) and epoxy-coated Ti6Al4V titanium alloy (B). 


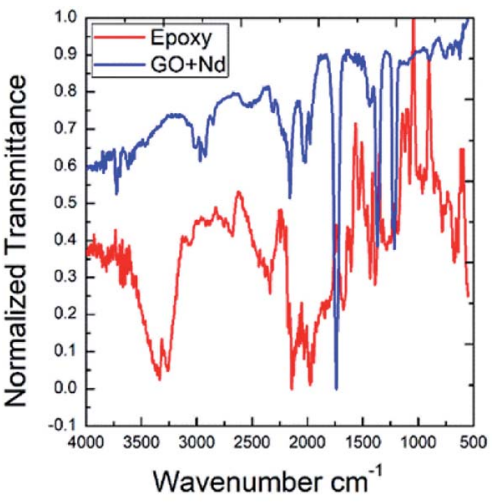

Fig. 12 ATR-FTIR normalized spectra of the epoxy and GO + Nd coated Ti6Al4V alloy surfaces after exposure to the corrosion conditions.

shown in Fig. 11, the epoxy coated titanium alloy surface exhibits pitting, and localized corrosion initiated due to the porosity of the barrier layer and aggressive ion attack. ${ }^{78,79}$ The atomic force microscopy images and roughness analysis (ESI, Fig. S2-S4†) show the effects of the penetration of aggressive ions into the epoxy layer, while confirming the corrosion inhibition of the GO + Nd coating..$^{\mathbf{8 0 1}}$

As shown in Fig. 12, the ATR-FTIR spectra after the exposure to the acidic environment of the epoxy and GO $+\mathrm{Nd}$ coatings onto the Ti6Al4V alloy was studied.

The epoxy coated alloy surface stretching frequency at $3500 \mathrm{~cm}^{-1}$ is associated with the hydroxyl groups adsorbed. ${ }^{82,83}$ The peak that appears at $2000 \mathrm{~cm}^{-1}$ is related to the alkyl chain vibration. The peaks at $1600 \mathrm{~cm}^{-1}$ and $1700 \mathrm{~cm}^{-1}$ could be attributed to the epoxy groups adsorbed on the alloy surface. In the spectrum of GO + Nd coating, peaks appear at $2900 \mathrm{~cm}^{-1}$ and $2800 \mathrm{~cm}^{-1}$ attributed to the $\mathrm{C}-\mathrm{C}, \mathrm{C}=\mathrm{C}$ graphite carbon chain, while the peak at $2300 \mathrm{~cm}^{-1}$ is associated with the $\mathrm{COOH}$ adsorbed on the alloy surface. ${ }^{\mathbf{8 4 , 8 5}}$ The peaks at $1388 \mathrm{~cm}^{-1}$ and $1720 \mathrm{~cm}^{-1}$ are attributed to $\mathrm{C}-\mathrm{O}$ and $\mathrm{C}=\mathrm{O}$, respectively. The peak at $920 \mathrm{~cm}^{-1}$ is associated with the Ti-O$\mathrm{Ti}$ bond vibration, indicating the possible formation of a $\mathrm{Ti}$ oxide surface layer.

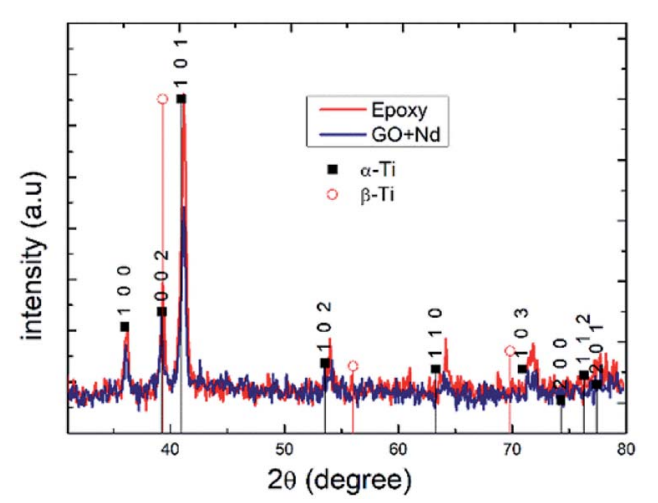

Fig. 13 X-ray diffractograms of epoxy and GO + Nd-coated Ti6Al4V alloy surfaces after exposure to corrosive media.

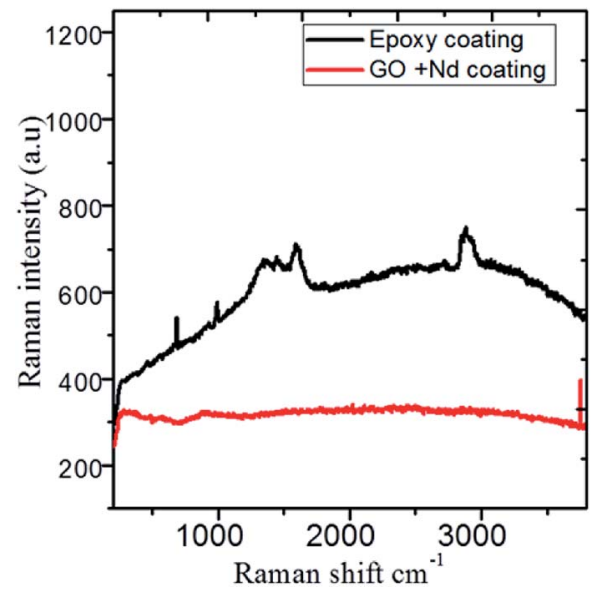

Fig. 14 Raman spectra of the epoxy and GO + Nd coated Ti6Al4V alloy surfaces after corrosion treatment.

Fig. 13 presents the X-ray diffractograms of the Ti6Al4Vcoated coupons after being exposed to the acidic environment to assess the possible presence of the corrosion products or structure modification due to aggressive ions diffusion. ${ }^{\mathbf{8 6 0}}$ The peaks in both diffractograms were indexed to the alfa Ti phase, and no clear presence of the beta-Ti phase, and none of $\mathrm{Ti}$ oxides or other corrosion products was found, although ATRFTIR results suggest the formation of $\mathrm{Ti}-\mathrm{O}-\mathrm{Ti}$ bond that could arise from surface $\mathrm{TiO}_{2} \cdot{ }^{91-95}$ The relatively higher peak intensities in the spectrum of epoxy reference coating arises from its lower thickness ( $c a .10$ micrometers) compared to that of the GO $+\mathrm{Nd}$ (ca. 14 micrometers).

The Raman spectra shown in Fig. 14 correspond to the epoxy and GO + Nd coated alloy surfaces after corrosion treatment. The alloy microstructure was investigated after 5 day immersion in the $1 \mathrm{M} \mathrm{H}_{2} \mathrm{SO}_{4}$ medium. The spectra of the epoxy coating on alloy surface has peaks at $410 \mathrm{~cm}^{-1}, 500 \mathrm{~cm}^{-1}, 640 \mathrm{~cm}^{-1}$ and $970 \mathrm{~cm}^{-1}$ related with the anatase phase, although due to the

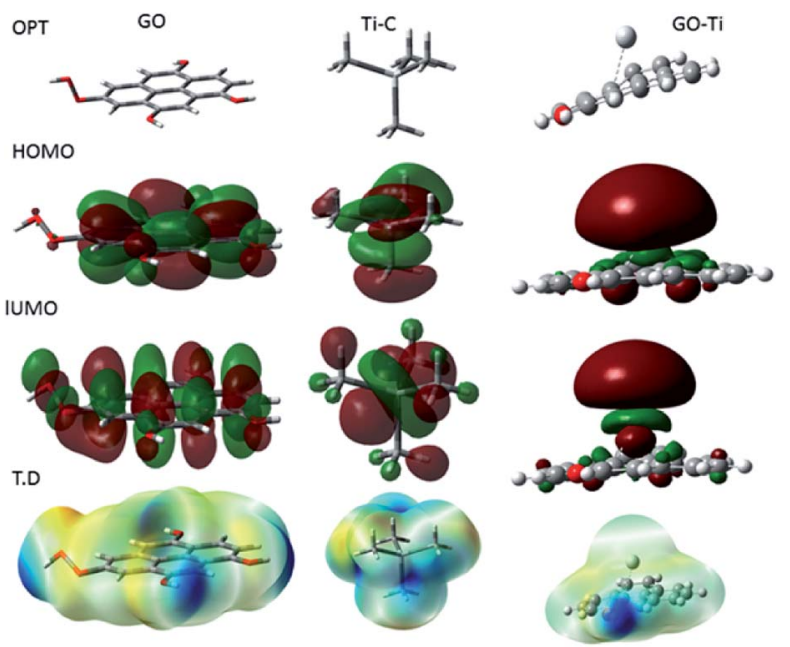

Fig. 15 Quantum chemical frontier molecular orbital structure of GO, $\mathrm{C}-\mathrm{Ti}$ and $\mathrm{GO}-\mathrm{Ti}$, and HOMO and LUMO electronic structures. 
Table 2 Quantum chemical values of GO and GO-Ti, model, rutile $\mathrm{TiO}_{2}$

\begin{tabular}{lllllll}
\hline S. no. & HOMO $(\mathrm{eV})$ & LUMO $(\mathrm{eV})$ & $\Delta E(\mathrm{eV})$ & $\chi(\mathrm{eV})$ & $\eta(\mathrm{eV})$ & $\sigma(\mathrm{eV})$ \\
\hline GO & 5.0327 & 1.3436 & 3.6894 & 3.1881 & 1.8445 & 0.5421 \\
Ti-C & 7.3236 & 1.8405 & 5.4831 & 4.5820 & 2.7415 & 0.3641 \\
GO-Ti & 3.3774 & 1.9518 & 1.4256 & 2.4015 & 0.7128 & 1.4256 \\
\hline
\end{tabular}

thickness, is not detected by XRD and is formed because the epoxy layer could not withstand the corrosive medium for a long time. The coating layer was ruptured due to the diffusion of the corrosion species in the alloy surface. ${ }^{96-100}$ The Raman spectrum of the GO + Nd-based coating onto the Ti6Al4V alloy shows the characteristic $\mathrm{D}$ and $\mathrm{G}$ band of graphene oxide, and has a very small evidence of the oxide layer with weak bands at $410 \mathrm{~cm}^{-1}$ and $970 \mathrm{~cm}^{-1}$, indicating the strong interaction of the $\mathrm{GO}+\mathrm{Nd}$ based coating with the alloy surface that prevents extensive corrosion.

\subsection{Quantum chemical studies}

Fig. 15 shows the DFT modelling of the GO onto a Ti surface. The graphene oxide model comprised 30 atoms, including one carboxyl group and three hydroxyl groups, and graphene oxide adsorption on titanium surface has been investigated for the possible electron transfer between the alloy surfaces and the inhibition barrier layer. ${ }^{\mathbf{1 0 1}, 102}$ The negatively charged GO surface favours the chemisorbed guest molecules on the plane surface. However, from the DFT studies, we suggest that neodymiummodified graphene oxide sheet is adsorbed on the alloy surface. Hence, the GO functional groups such as hydroxyl, carbonyl, and carboxyl could improve the chemisorption of the graphene oxide layer onto the Ti alloy. The electronic values of HOMO (highest unoccupied molecular orbital) is $c a .5 \mathrm{eV}$ and that of LUMO (lowest unoccupied molecular orbital) is $c a .1 .3 \mathrm{eV}$ for GO. The GO electronegativity, electrophilicity, and the softness and hardness values indicate a strong physisorption of the coating on the alloy surface, possibly because of the electron transfer between the coating surface and the polarized state of the metal surface. In the Ti-C model, the HOMO value increases to $7.32 \mathrm{eV}$, while the LUMO value increases to $1.84 \mathrm{eV}$, as well as the values for rest of the parameters except $\sigma$. With respect to the electron transfer between the graphene oxide sheet and the Ti oxide layer, the energy level of HOMO value decreases to $3.37 \mathrm{eV}$ while the LUMO energy level increases to $1.95 \mathrm{eV}$ compared with those of GO, suggesting that the titanium alloy could accept and donate electrons from the functionalized graphene oxide. ${ }^{103,104}$ The electronic parameters such as chemical hardness, softness, and electronegativity values contributed to the corrosion barrier activity on the alloys surface. The quantum chemical parameter values are presented in Table 2.

\section{Conclusions}

From the obtained results, the following conclusions can be stated: the $\mathrm{GO}+\mathrm{Nd}$ consists of $\mathrm{Nd}$ oxide nanorods in the hexagonal phase, attached to the GO sheet by the epoxy groups. The GO + Nd + epoxy coating onto the Ti6Al4V alloy showed a $65 \%$ increment in the corrosion inhibition activity with respect to the reference epoxy coating, as studied by the Tafel plots and the electrochemical impedance spectroscopy. The voltammetric analysis indicates that the $\mathrm{GO}+\mathrm{Nd}$ coating is stable and it can be regenerated even after voltage cycling in the acidic media. The Raman and ATR-FTIR spectra of the coated Ti6Al4V coupons after exposure to the acid medium suggested the formation of a $\mathrm{TiO}_{x}$ layer, which was not visible in XRD. The corresponding morphology analysis showed pit formation on the alloy with the epoxy coating, while GO + Nd coated surface was not affected by the aggressive ions, presenting a uniform surface. Finally, the modelling of the GO-Ti interactions showed the GO chemisorption on the alloy surface, confirming that $\mathrm{GO}+\mathrm{Nd}$ is an excellent barrier layer on titanium alloy in the acidic medium.

\section{Conflicts of interest}

There are no conflicts to declare.

\section{Acknowledgements}

NP would like to thank Central University of Gujarat Central Instrument Facility and Non-Net fellowship. NP would like to thank Professor M. V. Rajasekahran for the support provided for this work completion under the UGC Network Resource Centre Hyderabad, University of Hyderabad. FCB acknowledges financial support from SIP-IPN through 20194931 project. Professor K. R. Justin Thomas Department of Chemistry, Indian Institute of Technology Roorkee for supporting electron microscopy studies.

\section{References}

1 E. B. Tamarit, A. Igual-Munoz, J. G. Anton and D. M. GarciaGarcia, Corros. Sci., 2009, 5, 11095-11102.

2 C. S. Brossia and G. A. Cragnolino, Corros. Sci., 2004, 46, 1693-1711.

3 U. Eduok and J. Szpunar, Ultrason. Sonochem., 2018, 44, 288-298.

4 N. T. Kirkland, T. Schiller, N. Medhekar and N. Birbilis, Corros. Sci., 2012, 56, 1-4.

5 M. Breedon, M. C. Per, I. S. Cole and A. S. Barnard, J. Mater. Chem. A, 2014, 2, 16660-16668.

6 Y. N. Singhbabu, B. Sivakumar, J. K. Singh, H. Bapari, A. K. Pramanick and R. K. Sahu, Nanoscale, 2015, 7, 80358047.

7 D. Dutta, A. N. F. Ganda, J. K. Chih, C. C. Huang, C. J. Tsenga and C. Y. Su, Nanoscale, 2018, 10, 12612-12624. 
8 J. H. Huh, S. H. Kim, J. H. Chu, S. Y. Kim, J. H. Kim and S. Y. Kwon, Nanoscale, 2014, 6, 4379-4386.

9 F. Maia, J. Tedim, A. D. Lisenkov, A. N. Salak, M. L. Zheludkevich and M. G. S. Ferreira, Nanoscale, 2012, 4, 1287-1298.

10 C. Liu, S. Qiu, P. Du, H. Zhao and L. Wang, Nanoscale, 2018, 10, 8115-8124.

11 R. K. Gupta, M. Malviya, C. Verma, N. K. Gupta and M. A. Quraishi, RSC Adv., 2017, 7, 39063-39074.

12 Z. Qiu, R. Wang, J. Wu, Y. Zhang, Y. Qu and X. Wu, RSC Adv., 2015, 5, 44149-44159.

13 Y. Liu, J. Zhang, S. Li, Y. Wang, Z. Hana and L. Ren, RSC Adv., 2014, 4, 45389-45396.

14 Z. Yu, L. Lv, Y. Ma, H. Dia and Y. He, RSC Adv., 2016, 6, 18217-18226.

15 Y. K. Xiao, W. F. Ji, K. S. Chang, K. T. Hsu, J. M. Yeh and W. R. Liu, RSC Adv., 2017, 7, 33829-33836.

16 M. J. Nine, M. A. Cole, D. N. H. Tran and D. Losic, J. Mater. Chem. A, 2015, 3, 12580-12602.

17 Y. Deng, W. Bai, J. Chen, X. Zhang, S. Wang, J. Lin and Y. Xu, RSC Adv., 2017, 7, 45034-45044.

18 J. Di, S. Guo, L. Chen, P. Yi, G. Ding, K. Chen, M. Li, D. Lee and A. Yan, J. Rare Earths, 2018, 36, 826-831.

19 Z. Xichun, S. Xiaoyan and L. Zhongwu, J. Rare Earths, 2016, 34, 889.

20 Z. Qiaoying, L. Gang, L. Zhuang, G. Shuai, Y. Aru, L. Don and L. Jianzhong, J. Rare Earths, 2016, 34, 152.

21 Z. Dan, S. Jie, Z. Lili, T. Yong and L. Ji, J. Rare Earths, 2010, 28, 371-374.

22 Z. Enyao, C. Yungui and T. Yongbai, J. Rare Earths, 2012, 30, 269.

23 H. Jie, F. Song, H. Yan, L. Yi and X. Jianing, J. Rare Earths, 2016, 34, 283.

24 W. Daogao, Y. Shihong, W. Zhiqiang, Z. Zhiqi, M. Ruiying, Z. Xiaowei and C. Dehong, J. Rare Earths, 2014, 32, 663.

25 Y. Hongchuan, Z. Shirong, Y. Dunbo, L. Kuoshe, H. Quanxia, Y. Yuanfei, Z. Kun and L. Hongwei, J. Rare Earths, 2015, 33, 629.

26 B. Guangli, J. Jing, Z. Fan, F. Daqing, L. Yuandong, M. Ying and H. Yuan, J. Rare Earths, 2016, 34, 931.

27 Q. Yingjie, L. Wenpeng, W. Guixiang and Z. Xiaohong, J. Rare Earths, 2015, 33, 647.

28 Z. Mahidashti and B. Ramezanzadeh, J. Rare Earths, 2018, 36, 1112-1120.

29 H. Baojun, G. Dongdong, H. Qiong, Z. Xiaolian, P. Guanghuai and Y. Chubin, J. Rare Earths, 2016, 34, 731.

30 S. Ren, M. Cui, W. Li, J. Pu, Q. Xuea and L. Wang, J. Mater. Chem. A, 2018, 6, 24136-24148.

31 U. Eduok, O. Fayea and J. Szpunara, RSC Adv., 2016, 6, 108777-108790.

32 N. Palaniappan, I. S. Cole, F. C. Briones, K. Balasubaramanian and C. Lal, RSC Adv., 2018, 8, 34275-34286.

33 S. M. Derazkola, S. Z. Ajabshira and M. S. Niasar, RSC Adv., 2015, 5, 56666-56676.

34 M. Guzik, E. Tomaszewicz, Y. Guyot, J. Legendziewicza and G. Boulonc, J. Mater. Chem., 2012, 22, 14896-14906.
35 P. A. Bozkurt, Ultrason. Sonochem., 2017, 397-404.

36 B. Shahmoradi, K. Soga, S. Ananda, R. Somashekar and K. Byrappa, Nanoscale, 2010, 2, 1160-1164.

37 S. Anandana, A. M. Asiric and M. Ashokkumar, Ultrason. Sonochem., 2014, 21, 920-923.

38 J. Ma, Y. Gu, L. Shi, L. Chen and Y. Qian, Solid State Commun., 2004, 132, 743-745.

39 A. Hadia, J. Zahirifara, J. K. Sabet and A. Dastbaz, Ultrason. Sonochem., 2018, 44, 204-214.

40 B. Shahmoradi, K. Soga, S. Ananda, R. Somashekar and K. Byrappa, Nanoscale, 2010, 2, 1160-1164.

41 T. Saito, H. Sato and T. Motegi, J. Alloys Compd., 2006, 425, 145-147.

42 S. K. Ponnaiah, P. Prakash and B. Vellaichamy, Ultrason. Sonochem., 2018, 44, 196-203.

43 V. M. R. Betancourtt and D. Nattland, Phys. Chem. Chem. Phys., 2005, 77, 173-179.

44 V. Vinoth, J. J. Wu, A. M. Asiric and S. Anandan, Ultrason. Sonochem., 2017, 39, 363-373.

45 R. Chen, Y. Wang, Y. Liu and J. Li, RSC Adv., 2015, 5, 8506585072.

46 S. Sasikaladevi, J. Aravind, V. Eswaraiaha and S. Ramaprabhu, J. Mater. Chem., 2011, 21, 17094-17097.

47 D. M. Tobaldi, R. A. S. Ferreira, R. C. Pullar, M. P. Seabra, L. D. Carlos and J. A. Labrincha, J. Mater. Chem. C, 2015, 3, 4970-4986.

48 W. Yue, Z. Lin, S. Jiang and X. Yang, J. Mater. Chem., 2012, 22, 16318-16323.

49 A. Arunchander, M. Vivekanantha, S. G. Peera and A. K. Sahu, RSC Adv., 2016, 6, 95590-95600.

50 A. Dorris, C. Sicard, M. C. Chen, A. B. McDonald and C. J. Barrett, ACS Appl. Mater. Interfaces, 2011, 3, 3357-3365.

51 M. Kaplan, Z. Yuan, J. D. Benck, A. G. Rajan, X. S. Chu, Q. H. Wang and M. S. Strano, Chem. Soc. Rev., 2017, 46, 4530-4571.

52 C. Xu, B. Xu, Y. GU, Z. Xiong, J. Sun and X. S. Zhao, Energy Environ. Sci., 2013, 6, 1388-1414.

53 S. Szunerits and R. Boukherrou, Electrochemistry, 2014, 12, 211-242.

54 D. W. Chang, H. J. Choi, A. Filer and J. B. Baek, J. Mater. Chem. A, 2014, 2, 12136-12149.

55 M. T. Lee, C. Y. Fan, Y. C. Wang, H. Y. Li, J. K. Chang and C. M. Tseng, J. Mater. Chem. A, 2013, 1, 3395-3405.

56 N. Palaniappan, L. R. Chowhan, J. Sathiskumar, I. G. Bosco and I. S. Cole, Surf. Interfaces, 2017, 6, 237-246.

57 Y. Zhou, S. Xu, L. Guo, S. Zhang, H. Lu, Y. Gong and F. Gao, RSC Adv., 2015, 5, 14804-14813.

58 I. Tissot, O. C. Monteiro, M. A. Barreiros, V. Corregidor, J. Correia and M. F. Guerra, RSC Adv., 2016, 6, 51856-51863.

59 K. Fushimi, K. Kurauchi, Y. Yamamoto, T. Nakanishi, Y. Hasegawa and T. Ohtsuka, Electrochim. Acta, 2014, 144, 56-63.

60 D. Dutta, A. N. F. Ganda, J. K. Chih, C. C. Huang, C. J. Tseng and C. Y. Su, Nanoscale, 2018, 10, 12612-12624.

61 J. R. Chen and W. T. Tsai, Electrochim. Acta, 2011, 56, 17461751. 
62 F. Yu, L. Camilli, T. Wang, D. M. A. Mackenzie, M. Curioni, R. Akid and P. Boggild, Carbon, 2018, 132, 78-84.

63 Y. N. Singhbabu, B. Sivakumar, J. K. Singh, H. Bapari, A. K. Pramanick and R. K. Sahu, Nanoscale, 2015, 7, 80358047.

64 J. Hoon Huh, S. H. Kim, J. H. Chu, S. Y. Kim, J. H. Kim and S. Y. Kwon, Nanoscale, 2014, 6, 4379-4386.

65 S. K. Aneja, S. Bohm, A. S. Khanna and H. L. M. Bohm, Nanoscale, 2015, 7(7), 17879-17888.

66 J. H. Huh, S. H. Kim, J. H. Chu, S. Y. Kim, J. H. Kim and S. Y. Kwon, Nanoscale, 2014, 6, 4379-4386.

67 C. Liu, S. Qiu, P. Du, H. Zhao and L. Wang, Nanoscale, 2018, 10, 8115-8124.

68 E. Godlewska, M. Mitoraj and K. Leszczynska, Corros. Sci., 2014, 78, 63-70.

69 Z. B. Wang, H. X. Hu, Y. G. Zheng, W. Ke and Y. X. Qiao, Corros. Sci., 2016, 103, 50-65.

70 Y. Sia and Z. Guo, Nanoscale, 2015, 7, 5922-5946.

71 H. Lu, S. Zhang, W. Li, Y. Cui and T. Yang, ACS Appl. Mater. Interfaces, 2017, 9, 4034-4043.

72 W. Sun, L. Wang, T. Wu, M. Wang, Z. Yang, Y. Pan and G. Liu, Chem. Mater., 2015, 27, 2367-2373.

73 Y. P. Hsieh, M. Hofmann, K. W. Chang, J. G. Jhu, Y. Y. Li, K. Y. Chen, C. C. Yang, W. S. Chang and L. C. Chen, ACS Nano, 2014, 8, 443-448.

74 D. Prasai, J. C. Tuberquia, R. R. Harl, G. K. Jennings and K. I. Bolotin, ACS Nano, 2012, 6, 1102-1108.

75 A. M. Fekry, RSC Adv., 2016, 6, 20276.

76 N. Lin, Q. Liu, J. Zou, D. Li, S. Yuan, Z. Wang and B. Tang, RSC Adv., 2017, 7, 13517-13535.

77 M. A. Surmeneva, A. Vladescu, R. A. Surmenev, C. M. Pantilimon, M. Brai and C. M. Cotrut, RSC Adv., 2016, 6, 87665-87674.

78 L. Jinlong, Z. Huasheng and W. Yongxin, RSC Adv., 2016, 6, 105854-105861.

79 M. S. Hussein and A. M. Fekry, ACS Omega, 2019, 4, 73-78.

80 J. Rong Chen and W. Ta Tsai, Electrochim. Acta, 2011, 56, 1746-1751.

81 C. C. Hoa and S. J. Ding, J. Mater. Chem. B, 2015, 3, 2698.

82 V. Raj and M. S. Mumjitha, Electrochim. Acta, 2015, 153, 111.

83 B. Rikhari, S. Pugal Mani and N. Rajendran, $R S C A d v .$, 2016, 6, 80275-80285.

84 V. Jokanovic, M. VilotijevI, B. Jokanovic, M. Jenko, I. Anzel, D. Stamenkovic, V. Lazic and R. Rudolf, Corros. Sci., 2014, 82, 180-190.
85 A. C. Alves, F. Wenger, P. Ponthiaux, J. P. Celis, A. M. Pinto, L. A. Rocha and J. C. S. Fernandes, Electrochim. Acta, 2017, 234, 16-27.

86 A. U. Chaudhry, B. Mansoor, T. Mungole, G. Ayoub and D. P. Field, Electrochim. Acta, 2018, 264, 69-82.

87 T. M. Chiu, M. Mahmoudi, W. Dai, A. Elwany, H. Liang and H. Castaneda, Electrochim. Acta, 2018, 279, 143-151.

88 M. F. Lopez, J. A. Jimene and A. Gutierrez, Electrochim. Acta, 2003, 48, 1395-1401.

89 W. Simkaa, A. Sadkowski, M. Warczak, A. Iwaniak, G. Dercz, J. Michalska and A. Maciej, Electrochim. Acta, 2011, 56, 8962-8968.

90 J. Lin, S. Ozan, K. Munir, K. Wang, X. Tong, Y. Li, G. Li and C. Wen, RSC Adv., 2017, 7, 12309.

91 N. D. Alqarni, J. Wysocka, N. El-Bagoury, J. Ryl, M. A. Amin and R. Boukherroub, RSC Adv., 2018, 8, 19289-19300.

92 N. Lin, Q. Liu, J. Zou, D. Li, S. Yuan, Z. Wang and B. Tanga, RSC Adv., 2017, 7, 13517-13535.

93 B. Wua, Z. Pana, S. Lib, D. Cuiuria, D. Ding and H. Lia, Corros. Sci., 2018, 137, 176-183.

94 C. C. I. Popa, P. M. Brossard, D. Monceau and S. Chevalier, Corros. Sci., 2016, 110, 91-104.

95 V. Prabu, P. Karthick, A. Rajendran, D. Natarajan, M. S. Kiran and D. K. Pattanayak, RSC Adv., 2015, 5, 50767-50777.

96 P. Jianga, L. Lin, F. Zhanga, X. Dong, L. Rena and C. Lin, Electrochim. Acta, 2013, 107, 16-25.

97 L. Mohan, C. Anandan and N. Rajendran, Electrochim. Acta, 2015, 155, 411-420.

98 Z. J. Liua, X. Z. H. Liu, I.-L. Tsai, U. Donatus and G. E. Thompson, Electrochim. Acta, 2015, 182, 482-492.

99 E. Matykina, J. M. H. Lopez, A. Conde, C. Domingo, J. J. de Damborenea and M. A. Arenas, Electrochim. Acta, 2011, 56, 2221-2229.

100 D. W. Boukhvalov, RSC Adv., 2013, 3, 7150.

$101 \mathrm{~S}$. T. Skowron, I. V. Lebedeva, M. Popovic and E. Bichoutskaia, Chem. Soc. Rev., 2015, 44, 3143-3176.

102 S. Nigar, Z. Zhou, H. Wang and M. Imtiaze, RSC Adv., 2017, 7, 51546-51580.

103 Q. Tang, Z. Zhou and Z. Chen, Nanoscale, 2013, 5, 45414583.

104 M. Pykal, P. Jurecka, F. Karlickya and M. Otyepka, Phys. Chem. Chem. Phys., 2016, 18, 6351-6372. 